\title{
Instantaneous nonvertical electronic transitions with shaped femtosecond laser pulses: Is it possible?
}

\section{Henriksen, Niels Engholm; Møller, Klaus Braagaard}

Published in:

Journal of Chemical Physics

Link to article, DOI:

$10.1063 / 1.1586701$

Publication date:

2003

Document Version

Publisher's PDF, also known as Version of record

Link back to DTU Orbit

Citation (APA):

Henriksen, N. E., \& Møller, K. B. (2003). Instantaneous nonvertical electronic transitions with shaped femtosecond laser pulses: Is it possible? Journal of Chemical Physics, 119(5), 2569-2576.

https://doi.org/10.1063/1.1586701

\section{General rights}

Copyright and moral rights for the publications made accessible in the public portal are retained by the authors and/or other copyright owners and it is a condition of accessing publications that users recognise and abide by the legal requirements associated with these rights.

- Users may download and print one copy of any publication from the public portal for the purpose of private study or research.

- You may not further distribute the material or use it for any profit-making activity or commercial gain

- You may freely distribute the URL identifying the publication in the public portal

If you believe that this document breaches copyright please contact us providing details, and we will remove access to the work immediately and investigate your claim. 


\title{
Instantaneous nonvertical electronic transitions with shaped femtosecond laser pulses: Is it possible?
}

\author{
Niels E. Henriksen ${ }^{\text {a) }}$ and Klaus B. Møller \\ Department of Chemistry, Technical University of Denmark, DTU 207, DK-2800 Lyngby, Denmark
}

(Received 16 December 2002; accepted 5 May 2003)

\begin{abstract}
In molecular electronic transitions, a vertical transition can be induced by an ultrashort laser pulse. That is, a replica of the initial nuclear state-times the transition dipole moment of the electronic transition-can be created instantaneously (on the time scale of nuclear motion) in the excited electronic state. Now, applying pulse shaping via the modulation of the phases of each spectral component of an ultrashort pulse, it is tempting to ask whether it is also possible to induce instantaneous nonvertical transitions to bound electronic states, provided that the phases of each spectral component of the pulse are set to appropriate values at the discrete frequencies corresponding to the energy levels of the potential. We analyze the problem in the weak-field limit, and show that such a phase requirement cannot be encoded into an ultrashort pulse. This result is equivalent to the statement that it is not possible to move matter faster than the time associated with the natural (field-free) dynamics of the system. (C) 2003 American Institute of Physics.
\end{abstract}

[DOI: $10.1063 / 1.1586701]$

\section{INTRODUCTION}

Short laser pulses can be used to steer molecular dynamics including chemical reactions. ${ }^{1-3}$ Given some initial state, the aim of laser control is to guide a system into a desired final state. To that end, laser fields with complex pulse shapes can now be created experimentally. Thus it is, e.g., possible to modulate the phases of each spectral component of a short pulse. ${ }^{4}$ Furthermore, using the concept of laboratory feedback control, ${ }^{5}$ it is possible to obtain control without any prior knowledge of the system Hamiltonian. This procedure is based on the feedback from an observed experimental signal and an algorithm that iteratively improves the applied femtosecond laser pulse. Several applications of this approach have been published recently. ${ }^{6-11}$

At least in simple systems like atoms ${ }^{12}$ and small molecules ${ }^{13,14}$ theory can be used to guide the choice of pulse shape as well as a tool for interpreting the resulting complex pulse shape, i.e., how the field induces a given desired result. Here we focus on (weak-field) molecular electronic transitions and in this case it is a well-known theoretical result, that an infinitely short pulse-a so-called $\delta(t)$ pulse-induces a vertical transition. That is, a FranckCondon wave packet, which is a replica of the initial nuclear state times the transition dipole moment of the electronic transition, is created in the excited electronic state, ${ }^{15,16}$ see Fig. 1(a). This is the limiting case of the general result that an ultrashort (femtosecond) pulse creates a nuclear wave packet in the excited electronic state. The idea of pulse shaping is to control the relative amplitudes and phases of the vibronic eigenstates that constitute this wave packet and thereby its shape and/or location through quantum interference. In the weak-field limit, the relative amplitudes and

\footnotetext{
a) Author to whom correspondence should be addressed. Electronic mail: neh@kemi.dtu.dk; Fax (+45) 45883136.
}

phases of the various components of the excited-state wave packet are in fact given directly by the relative amplitudes and phases of the corresponding frequencies within the driving field, which in turn are experimentally controllable quantities. 4

In this paper we wish to provide some additional insight into the dynamics induced by ultrashort shaped laser pulses and discuss possible limitations on what targets can be obtained-exemplified by the question: is it possible to construct an ultrashort laser pulse that can induce an instantaneous nonvertical electronic transition in a molecule? Before we go on, we must qualify the statement "instantaneous nonvertical transition" and why it is a good prototype example. Figure 1 illustrates what we mean by nonvertical transition, namely the creation of a wave packet similar to the FranckCondon packet except for its location. Due to the energetics of this setup, it is reasonable to expect that the pulse needed to create the situation in panel (b) may be obtained from the pulse creating the situation in panel (a) by pulse shaping. In fact the situation depicted in Fig. 1 is particularly simple since the potentials are considered harmonic and therefore the expansion coefficients onto eigenstates of the excitedstate wave functions in panels (a) and (b) differ only by a sign. For the same reason, however, the wave function in panel (a) will evolve into the wave function in panel (b) after half a vibrational period (and vice versa). Hence, in the case of a $\delta(t)$-pulse excitation, it will take half a vibrational period for the promoted state to evolve into the desired target state and the problem is trivial. The question we ask is therefore whether with an ultrashort shaped laser pulse one can "beat" this time associated with the natural dynamics. Thus we are going consider whether the average bond lengths can change very fast compared to the time scale of nuclear motion in a molecule. That is, right after a femtosecond excitation (say corresponding to less than $1 / 10$ of a vibrational 
a

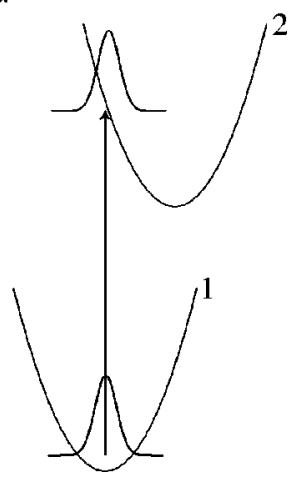

b

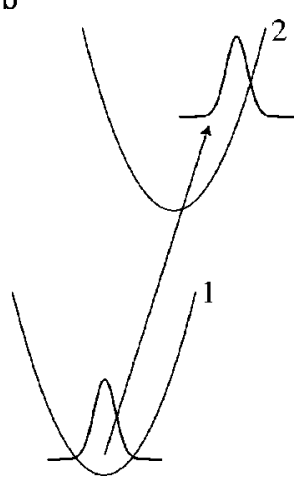

FIG. 1. Vertical (a) and nonvertical (b) electronic transitions induced by ultrashort laser pulses. Electronic energies are represented by displaced harmonic potentials. The initial state is the vibrational ground state. The transition dipole moment of the electronic transition is assumed to be constant (chosen as 1). Note that the baselines of the wave packets are drawn such that they reflect the expectation value of the energy. In the excited state the energy is $(1 / 2) m \omega^{2} d^{2}+(1 / 2) \hbar \omega$, where $\omega$ is the frequency and $d$ is the displacement of the two harmonic potentials.

period) the average distances might have changed by the order of $1 \AA$, equivalent to a speed of $v$ $=10^{-10} \mathrm{~m} /\left(10^{-15} \mathrm{~s}\right)=10^{5} \mathrm{~m} / \mathrm{s}$.

The present work is closely related to recent theoretical work on the construction of laser fields that will generate a desired target vibrational wave packet in a diatomic molecule. ${ }^{14}$ In that work, laser fields of shorter duration than the characteristic time scale of the system were explicitly considered. Our objective is also related to the recent experimental work on time shift of wave packets. ${ }^{17}$ It was demonstrated that laser phases can be tailored such that at early times a wave packet is created that otherwise only would be obtained at longer times due to the natural dynamics of the system. We will consider these works in more detail in the following sections.

In the next section, we review the theoretical framework needed in order to describe the wave packet created by a laser pulse where the amplitude as well as the phase of each spectral component is specified. In Sec. III, we present some analytical expressions for the time-dependent electric field of the laser pulse for various representations of the required phase function. We focus on the phase function required in order to create a copy of the initial nuclear state (times the transition dipole moment of the electronic transition) at the outer turning point of a symmetric excited state potential. Finally, conclusions are given in Sec. IV.

\section{THEORY}

We consider an electronic transition in a molecule, from electronic state " 1 " to state " 2 ." Within the electric-dipole approximation and first-order perturbation theory for the interaction with an electromagnetic field, the state vector associated with the nuclear motion in electronic state 2 is given by (for times $t$, where the laser pulse has vanished) ${ }^{16,18,19}$

$$
\left|\chi_{2}(t)\right\rangle=\exp \left(-i \hat{H}_{2} t / \hbar\right)\left|\chi_{2}\right\rangle \text {. }
$$

Here, $\left|\chi_{2}\right\rangle$ is the promoted state,

$$
\left|\chi_{2}\right\rangle=\frac{i}{\hbar} \int_{-\infty}^{\infty} d t^{\prime} e^{-i \epsilon_{0} t^{\prime} / \hbar} E\left(t^{\prime}\right) \exp \left(i \hat{H}_{2} t^{\prime} / \hbar\right)|\phi\rangle,
$$

where $E(t)$ is the laser field and

$$
|\phi\rangle=\mu_{12}\left|\chi_{1}\right\rangle
$$

is the Franck-Condon wave packet with $\left|\chi_{1}\right\rangle$ being the initial stationary nuclear state in electronic state 1 with energy $\epsilon_{0}$, and $\mu_{12}$ is the projection of the electronic transition dipole moment on the polarization vector of the electric field.

The time-evolution operator in Eqs. (1) and (2) can be written in the form

$$
\exp \left(-i \hat{H}_{2} t / \hbar\right)=\sum_{n}\left|E_{n}\right\rangle e^{-i E_{n} t / \hbar}\left\langle E_{n}\right|,
$$

where $\left|E_{n}\right\rangle$ is the stationary state

$$
\hat{H}_{2}\left|E_{n}\right\rangle=E_{n}\left|E_{n}\right\rangle
$$

for an excited-state Hamiltonian with a purely discrete spectrum. From Eqs. (2) and (4)

$$
\left|\chi_{2}\right\rangle=\frac{i}{\hbar} \sum_{n}\left\langle E_{n} \mid \phi\right\rangle\left|E_{n}\right\rangle F\left(E_{n}\right),
$$

where

$$
F\left(E_{n}\right)=\int_{-\infty}^{\infty} d t^{\prime} e^{-i\left(\epsilon_{0}-E_{n}\right) t^{\prime} / \hbar} E\left(t^{\prime}\right)
$$

is the Fourier transform of the field, at the energies associated with the nuclear states of the upper electronic state.

The electric field can be written in the form

$$
E(t)=E_{0} \operatorname{Re}\left\{\int_{-\infty}^{\infty} A\left(\omega_{l}\right) e^{i \phi\left(\omega_{l}\right)} e^{-i \omega_{l} t} d \omega_{l}\right\},
$$

where $A\left(\omega_{l}\right)$ is the real-valued distribution of frequency components and $\phi\left(\omega_{l}\right)$ is the real-valued frequencydependent phase. For simplicity, we consider in the following only the dimensionless electric field defined by $\mathcal{E}(t)$ $\equiv E(t) / E_{0}$.

Now, with $\omega_{E_{n}}=\left(E_{n}-\epsilon_{0}\right) / \hbar$, Eq. (7) takes the form

$$
\begin{aligned}
F\left(E_{n}\right)= & \int_{-\infty}^{\infty} d t^{\prime} e^{i \omega_{E_{n}} t^{\prime}} \mathcal{E}\left(t^{\prime}\right) \\
= & \frac{1}{2} \int_{-\infty}^{\infty} \int_{-\infty}^{\infty}\left\{A\left(\omega_{l}\right) e^{i \phi\left(\omega_{l}\right)} e^{i\left(\omega_{E_{n}}-\omega_{l}\right) t^{\prime}}\right. \\
& \left.+A\left(\omega_{l}\right) e^{-i \phi\left(\omega_{l}\right)} e^{i\left(\omega_{E_{n}}+\omega_{l}\right) t^{\prime}}\right\} d t^{\prime} d \omega_{l} \\
= & \pi \int_{-\infty}^{\infty}\left\{A\left(\omega_{l}\right) e^{i \phi\left(\omega_{l}\right)} \delta\left(\omega_{E_{n}}-\omega_{l}\right)\right. \\
& \left.+A\left(\omega_{l}\right) e^{-i \phi\left(\omega_{l}\right)} \delta\left(\omega_{E_{n}}+\omega_{l}\right)\right\} d \omega_{l} \\
= & \pi A\left(\omega_{E_{n}}\right) e^{i \phi\left(\omega_{E_{n}}\right)}+\pi A\left(-\omega_{E_{n}}\right) e^{-i \phi\left(-\omega_{E_{n}}\right) .}
\end{aligned}
$$

The last term is insignificant because the pulse can be represented solely in terms of positive frequencies. That is,

$$
\left|\chi_{2}\right\rangle=\frac{i \pi}{\hbar} \sum_{n} A\left(\omega_{E_{n}}\right) e^{i \phi\left(\omega_{E_{n}}\right)}\left\langle E_{n} \mid \phi\right\rangle\left|E_{n}\right\rangle .
$$


Thus for a bound-bound transition, the promoted state takes the form

$$
\left|\chi_{2}\right\rangle=\frac{i \pi}{\hbar} \sum_{n} C_{n}\left|E_{n}\right\rangle
$$

where

$$
C_{n}=A\left(\omega_{E_{n}}\right)\left\langle E_{n} \mid \phi\right\rangle e^{i \phi\left(\omega_{E_{n}}\right)}
$$

and $\left|E_{n}\right\rangle$ is a nuclear eigenstate of electronic state 2. Note that only the amplitude and phase values of the field at the discrete and specific excitation frequencies $\omega_{E_{n}}=\left(E_{n}\right.$ $\left.-\epsilon_{0}\right) / \hbar$ affect the wave packet. For future reference, we denote by $\left|\chi_{2}\right\rangle_{0}$ the promoted state created by a bandwidthlimited pulse, that is, a field with the phase function $\phi\left(\omega_{l}\right)$ $=0$.

Thus the phase of each spectral component of the field translates directly into the phase of each expansion coefficient of the promoted state, and a particular phase relation between the expansion coefficients can accordingly be created experimentally by the laser pulse. Note that, in principle, any wave packet can be created because $\left|E_{n}\right\rangle$ forms a complete set of states and the expansion coefficients contain the complex valued spectral components of the field which, in principle, are under complete experimental control. However, in practice we are limited by the bandwidth of the laser, and furthermore it will, e.g., be difficult to create substantial amplitude in eigenstates where the associated FranckCondon factor $\left\langle E_{n} \mid \phi\right\rangle$ is very small (for complementary work on the control of non-Franck-Condon transitions, see Ref. 20).

As an example, the choice $\phi\left(\omega_{E_{n}}\right)=-E_{n} t_{\text {shift }} / \hbar$ results in a time shift of the packet with respect to the one created by the corresponding bandwidth-limited $\left[\phi\left(\omega_{l}\right)=0\right]$ laser pulse. ${ }^{17}$ That is, the phases can be tailored such that at early times a wave packet is created that otherwise only would be obtained at longer times ( $t_{\text {shift }}$ later) due to the natural dynamics of the system subsequent to excitation by a bandwidth-limited laser pulse,

$$
\begin{aligned}
\left|\chi_{2}(t)\right\rangle= & \exp \left(-i \hat{H}_{2} t / \hbar\right) \frac{i \pi}{\hbar} \sum_{n} A\left(\omega_{E_{n}}\right) \\
& \times\left\langle E_{n} \mid \phi\right\rangle e^{-i E_{n} t_{\text {shift }} / \hbar}\left|E_{n}\right\rangle \\
= & \exp \left[-i \hat{H}_{2}\left(t+t_{\text {shift }}\right) / \hbar\right]\left|\chi_{2}\right\rangle_{0} .
\end{aligned}
$$

We can, e.g., imagine creating-instantaneously —a packet at the "outer" turning point of a bound potential using an ultrashort pulse. (Here, and in the following, we assume that the equilibrium distance on electronic state 2 is larger than the equilibrium distance on electronic state 1 such that the Franck-Condon wave packet is created at the "inner" turning point on electronic state 2 and subsequently moves outwards. The outer turning point is then defined as the point where the wave packet turns around and starts to move inwards. If the equilibrium distance on electronic state 2 is shorter than the equilibrium distance on electronic state 1 , the designation of inner and outer is reversed.) This could be achieved in a setup (if possible) where the pulse is only nonvanishing for very short times $t<t_{\mathrm{f}}$ (on the time scale of nuclear motion) such that right after the pulse has vanished, we have

$$
\left|\chi_{2}\left(t_{\mathrm{f}}\right)\right\rangle=\exp \left(-i \hat{H}_{2} t_{\mathrm{f}} / \hbar\right)\left|\chi_{2}\right\rangle \approx\left|\chi_{2}\right\rangle
$$

and the phase function is chosen as specified above with $t_{\text {shift }}=P / 2$, where $P / 2$ is the time it takes to reach the outer turning point (half a vibrational period in a harmonic potential). This corresponds exactly to the picture in Fig. 1, where the excited-state potential is harmonic. However, in general, the time-evolved Franck-Condon wave packet may have changed form when it reaches the outer turning point.

Note that the phase function introduced above only is defined at discrete and specific frequencies. It is, however, very important to notice that a $\delta(t)$ pulse is expected only when $A\left(\omega_{l}\right)$ as well as the phases $\phi\left(\omega_{l}\right)$ are constant. An essential question in the following is now: Can one create a shaped pump pulse-with the required specific phase values - which is nonvanishing only for times $\ll$ than (half) a vibrational period?

To address this issue, our strategy is the following: (i) assume that the pulse is sufficiently short to make Eq. (14) a reasonably good approximation, (ii) determine from Eq. (12) the field to comply with the target $\left|\chi_{2}\right\rangle$, and (iii) calculate $\mathcal{E}(t)$ using Eq. (8) and check whether it satisfies the assumption made in (i).

Since the phase function introduced above is defined only at discrete and specific frequencies, one could anticipate that it can be implemented without (noticeable) changes in $\mathcal{E}(t)$ compared to the corresponding bandwidth-limited field. Thus, if we consider a short pulse and change the phase function only at isolated frequencies, the change in the Fourier integral of Eq. (8) might turn out to be limited.

As an alternative to the "time-shifting" approach, consider the choice ${ }^{14}$

$$
A\left(\omega_{l}\right) e^{i \phi\left(\omega_{l}\right)}=\sum_{n} \frac{C_{n}}{\left\langle E_{n} \mid \phi\right\rangle} \delta\left(\omega_{l}-\omega_{E_{n}}\right),
$$

where the field is nonzero only at the required frequencies and in agreement with Eq. (12). Then according to Eq. (8), this choice leads to the electric field

$$
\mathcal{E}(t)=\operatorname{Re}\left\{\sum_{n} \frac{C_{n}}{\left\langle E_{n} \mid \phi\right\rangle} e^{-i \omega_{E_{n}}{ }^{t}}\right\} .
$$

This field takes generally the form of a series of peaks spaced by the characteristic time scale of the system. The duration of the overall pulse is certainly much longer than requested, but it has been suggested that any of the subimpulses can generate the desired target state. ${ }^{14}$ We will consider these propositions in the following sections.

\section{DISPLACED HARMONIC OSCILLATORS: SYMMETRIC POTENTIALS}

In order to illustrate the basic concepts in greater detail, we consider now two electronic energy states within a harmonic approximation, that is, for small displacements around the equilibrium positions. With the objective of nonvertical transitions, it is relevant to consider the case where the ex- 
pansion coefficients $C_{n}$ in Eq. (11) are chosen such that they coincide with the expansion coefficients of a coherent state.

We note that $\left\langle E_{n} \mid \phi\right\rangle$, within the Condon approximation, are proportional to the overlaps (Franck-Condon factors) of the initial state with the eigenstates of a (displaced) harmonic oscillator. When the initial state is the vibrational ground state, then for displaced harmonic oscillators with identical force constants, $\left\langle E_{n} \mid \phi\right\rangle=\mu_{21} c_{n}$ where ${ }^{21,22}$

$$
c_{n}=(-1)^{n} e^{-(1 / 2)|\alpha|^{2}} \frac{|\alpha|^{n}}{\sqrt{n !}}
$$

are the expansion coefficients of a coherent state with

$$
|\alpha|^{2}=m \omega d^{2} /(2 \hbar),
$$

where $d$ is the displacement and $m \omega=\sqrt{m k}$, where $k$ is the force constant (vibrational period $P=2 \pi / \omega$ ). Note that the phase in Eq. (17) is chosen in accordance with the usual sign convention $^{21}$ and the assumption that the equilibrium distance on electronic state 2 is larger than the equilibrium distance on electronic state 1, i.e., $V_{2}(r)=V_{1}(r-d)$, cf. Fig. 1 . In this scenario, a coherent state at the outer turning point (our target) has the expansion coefficients, $(-1)^{n} c_{n}$. The probability distribution $\left|c_{n}\right|^{2}=\exp \left(-|\alpha|^{2}\right)|\alpha|^{2 n} / n$ ! corresponds to a Poisson distribution (average value as well as the variance of $n$ given by $\bar{n}=|\alpha|^{2}$ ).

For a bandwidth-limited pulse where $A\left(\omega_{l}\right)$ is constant over the width of the Poisson distribution-corresponding to an ultrashort pulse-we have $C_{n}=\left\langle E_{n} \mid \phi\right\rangle$. That is, the promoted state equals the Franck-Condon wave packet. Note that as the variance $|\alpha|^{2} \propto d^{2}$ (i.e., the displacement) is increased, the pulse must be shorter and shorter in order to reach this "ultrashort-pulse" limit.

For an ultrashort shaped pulse, the phase of the field determines the phase of $C_{n}, \arg \left(C_{n}\right)=\arg \left(c_{n}\right)+\phi\left(\omega_{E_{n}}\right)$, and therefore the position of the wave packet subsequent to the excitation. It is clear that, in the present case, any phase function satisfying the relation, $\phi\left(\omega_{E_{n}}\right)= \pm n \pi+$ const, would create the desired wave packet at the outer turning point. Note that for the harmonic excited-state potential this is equivalent to the time shift mentioned above, since $\phi\left(\omega_{E_{n}}\right)=-E_{n} t_{\text {shift }} / \hbar=-n \pi+$ const, for $E_{n}=\hbar \omega n+$ const and $t_{\text {shift }}=P / 2=\pi / \omega$.

In general, an ultrafast pulse with a phase function satisfying the relation $\phi\left(\omega_{E_{n}}\right)= \pm n \pi+$ const would create a "mirror image" of the Franck-Condon wave packet at the outer turning point on any symmetric excited-state potential.

\section{A. Various representations of the phase function}

In this section, we present a theoretical investigation of the electric field associated with various phase functions $\phi\left(\omega_{l}\right)$. Note that since we only have to fix the phase at some discrete and specific frequencies, we can choose arbitrary phases at all frequencies except at the specific frequencies. The goal is to choose the arbitrary phase values in the phase function such that the pulse is nonzero only for times much smaller than (half) a vibrational period.

We consider in the following some representations of the phase function which all fulfill the phase condition specified above. The electric field $\mathcal{E}(t)$ is then reconstructed according to Eq. (8). Numerical illustrations are for the abovementioned harmonic-oscillator system such that $\omega_{E_{n}}=\left(E_{n}\right.$ $\left.-\epsilon_{0}\right) / \hbar=n \omega+\Omega_{00}$, where $\Omega_{00}=\left(E_{0}-\epsilon_{0}\right) / \hbar$ is the 00 transition frequency.

First, we consider the electric field of Eq. (16). From the discussion above, we obtain the following driving field for the Franck-Condon wave packet $\left(C_{n}=\left\langle E_{n} \mid \phi\right\rangle\right)$,

$$
\begin{aligned}
\mathcal{E}_{\mathrm{FC}}(t)= & \operatorname{Re}\left\{\sum_{n=0}^{\infty} e^{-i \omega_{E_{n}} t}\right\}=\operatorname{Re}\left\{e^{-i \Omega_{00} t} \sum_{n=0}^{\infty} e^{-i n \omega t}\right\} \\
= & \operatorname{Re}\left\{e^{-i \Omega_{00} t} \frac{1}{\omega} \sum_{n=-\infty}^{\infty}[\pi \delta(t-2 n \pi / \omega)\right. \\
& \left.\left.+i \mathcal{P}\left(\frac{1}{t-2 n \pi / \omega}\right)\right]\right\},
\end{aligned}
$$

where $\mathcal{P}$ denotes a Cauchy principal value. ${ }^{21}$ Thus a series of identical (apart from a scaling) ultrashort $\delta$ pulses at $t=0$, $\pm P, \pm 2 P, \ldots$ The field which generates a copy of the Franck-Condon wave packet at the outer turning point $\left(C_{n}\right.$ $\left.=(-1)^{n}\left\langle E_{n} \mid \phi\right\rangle\right)$ is

$$
\begin{aligned}
\mathcal{E}(t)= & \operatorname{Re}\left\{\sum_{n=0}^{\infty}(-1)^{n} e^{-i \omega_{E_{n}} t}\right\} \\
= & \operatorname{Re}\left\{e^{\left.-i \Omega_{00^{t}} \sum_{n=0}^{\infty} e^{-i n(\omega t-\pi)}\right\}}\right. \\
= & \operatorname{Re}\left\{e^{-i \Omega_{00} t} \frac{1}{\omega} \sum_{n=-\infty}^{\infty}[\pi \delta(t-m \pi / \omega)\right. \\
& \left.\left.+i \mathcal{P}\left(\frac{1}{t-m \pi / \omega}\right)\right]\right\},
\end{aligned}
$$

where $m=2 n+1$. Thus this field is identical in nature to the previous field, except that the individual peaks have been shifted by half a vibrational period (this relation between the two fields is also true for a finite number of terms in the sum over bound states). The shift by half a period seems natural bearing in mind that it takes exactly half a period for the Franck-Condon packet to reach the outer turning point (cf. the discussion below concerning a linear phase function). Of course, the above fields do not fulfill our requirement of being ultrashort. To this end, it has been proposed ${ }^{14}$ that in a multipeak structure like this, one might be able to pick just a single of these impulses, shift it to time zero, and use it as the driving field to generate the desired target state. If the individual impulses are sufficiently short, as they obviously are in this case, the driving field obtained this way would comply with our objectives. Clearly, this procedure works for Eq. (19), since any ultrashort $\delta$ pulse creates the Franck-Condon wave packet right after the pulse has vanished. However, for that same reason it is not possible to apply this procedure to the field in Eq. (20) in order to obtain a copy of the FranckCondon wave packet at the outer turning point right after the driving field has vanished.

Therefore we continue to investigate other types of fields with the required phase relation. In the following, we start 
with a bandwidth-limited pulse and then modulate the phases of each spectral component, exactly as in the experimental approach to laser-pulse shaping. ${ }^{4}$

\section{Polynomial phase functions}

As an example, consider a Gaussian frequency distribution (centered at $\omega_{0}$ ) with a quadratic phase function, i.e.,

$$
A\left(\omega_{l}\right) e^{i \phi\left(\omega_{l}\right)}=\sqrt{\frac{a}{\pi}} \exp \left[-a\left(\omega_{l}-\omega_{0}\right)^{2}\right] e^{i\left(b \tilde{\omega}_{l}+c \tilde{\omega}_{l}^{2}\right)},
$$

where $\widetilde{\omega}_{l}=\omega_{l}-\Omega_{00}$. The electric field then takes the form

$$
\begin{aligned}
\mathcal{E}(t)= & e^{-\left[\alpha \tau^{2}-4 c \Delta(\alpha \tau-a \beta \Delta)\right]} \\
& \times \operatorname{Re}\left\{\sqrt{\frac{a}{a-i c}} e^{-i\left[\beta \tau^{2}+4 a \Delta(\alpha \tau-a \beta \Delta)+\Omega_{00} t\right]}\right\},
\end{aligned}
$$

where $\Delta=\omega_{0}-\Omega_{00}$ is the detuning of the laser from the 00 transition, $\quad \tau=t-b, \quad \alpha=a /\left(4 a^{2}+4 c^{2}\right), \quad$ and $\beta=c /\left(4 a^{2}\right.$ $\left.+4 c^{2}\right)$. Note that the pulse is broadened by the quadratic part of the phase function determined by the $c$ parameter. The pulse is, however, just shifted in time by the linear part of the phase function, determined by the $b$ parameter. These results are, of course, well known. For $b=\pi / \omega$ and $c=0$, we get the required values of the phase function and a pulse, $\mathcal{E}(t)=\exp \left[-(t-\pi / \omega)^{2} /(4 a)\right] \cos \left(\omega_{0} t-\Delta \pi / \omega\right)$, which is shifted in time by half a vibrational period. This result is easy to interpret. The promoted state for such an ultrashort pulse, $\left|\chi_{2}\right\rangle \propto \exp \left[i \hat{H}_{2}(P / 2) / \hbar\right]|\phi\rangle$, is exactly the desired target state: a copy of the Franck-Condon wave packet at the outer turning point of the potential. However, the actual wave packet right after the pulse has vanished, $\left|\chi_{2}\left(t_{\mathrm{f}}\right)\right\rangle \approx\left|\chi_{2}(P / 2)\right\rangle$ $=\exp \left[-i \hat{H}_{2}(P / 2) / \hbar\right]\left|\chi_{2}\right\rangle$, is equal (proportional) to the Franck-Condon wave packet. This is, of course, the result we would expect for an ultrashort pulse, irrespective of the time where it is fired. For $b=0$ and $c=\pi / \omega^{2}$, we get again values of the phase which will give the required alternating signs of $\exp \left[i \phi\left(\omega_{l}\right)\right]$. For $a \omega^{2}=0.0355971$, corresponding to a (bandwidth-limited) Gaussian pulse where the pulse duration [full width at half maximum (FWHM)] is $1 / 10$ of the vibrational period, the pulse is broadened significantly. The temporal width (FWHM) is now $\sim 8.8$ vibrational periods and, again, not useful for our purpose.

The electric field corresponding to a Gaussian frequency distribution with a cubic phase function can also be evaluated analytically,

$$
A\left(\omega_{l}\right) e^{i \phi\left(\omega_{l}\right)}=\sqrt{\frac{a}{\pi}} \exp \left[-a\left(\omega_{l}-\omega_{0}\right)^{2}\right] e^{i \pi\left(\tilde{\omega}_{l} / \omega\right)^{3}} .
$$

The electric field takes then the form (for simplicity, $\omega_{0}$ $\left.=\Omega_{00}\right)$

$$
\begin{aligned}
\mathcal{E}(t)= & 2 \alpha \sqrt{\pi\left(a \omega^{2}\right)} \exp \left(\frac{2\left(a \omega^{2}\right)^{3}}{27 \pi^{2}}-\frac{a \omega^{2}}{3 \pi} \omega t\right) \\
& \times \operatorname{Ai}\left[\alpha\left(\frac{\left(a \omega^{2}\right)^{2}}{3 \pi}-\omega t\right)\right] \cos \left(\omega_{0} t\right),
\end{aligned}
$$

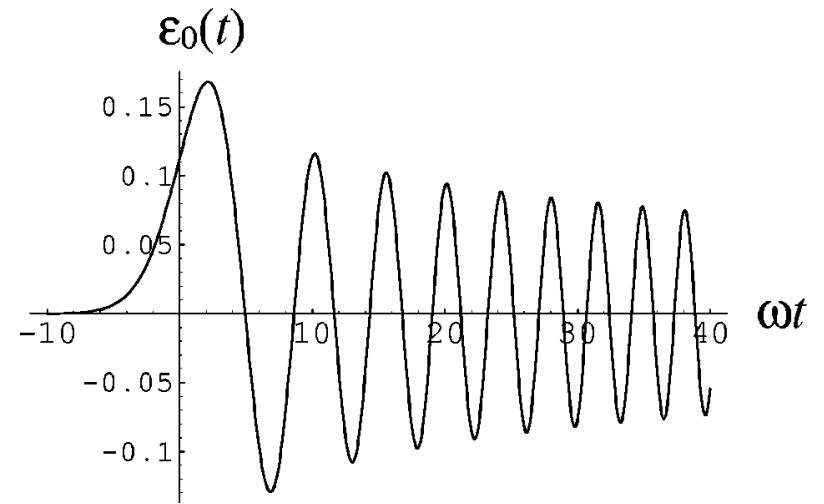

FIG. 2. The envelope of the electric field $\left[\mathcal{E}_{0}(t)=\mathcal{E}(t) / \cos \left(\omega_{0} t\right)\right]$ for a Gaussian frequency distribution with a cubic phase function, see Eq. (24). The temporal width (FWHM) of the transform limited Gaussian distribution is $\omega t=\pi / 5$. Note that $\omega t=2 \pi$ corresponds to one vibrational period.

where $\alpha=(3 \pi)^{-1 / 3}$ and $\operatorname{Ai}(x)$ is the Airy function.

Figure 2 shows $\mathcal{E}_{0}(t)=\mathcal{E}(t) / \cos \left(\omega_{0} t\right)$ for $a \omega^{2}$ $=0.0355971$, corresponding to a (bandwidth-limited) Gaussian pulse where the pulse duration (FWHM) is $1 / 10$ of the vibrational period. Clearly, the introduction of the cubic phase function broadens the pulse significantly, such that the pulse duration now is substantially longer than one vibrational period. This result is again very far from the desired result!

\section{Phase functions with constant segments}

The required phases can, e.g., be fixed in some intervals around the energy eigenstates:

$$
\phi\left(\omega_{l}\right)= \begin{cases}\pi & \text { if }\left|\omega_{l}-\omega_{E_{n}}\right|<\varepsilon_{n}, \quad n=1,3,5, \ldots \\ 0 & \text { otherwise }\end{cases}
$$

where the widths of the segments are $\varepsilon_{n}<\omega$.

Assuming that $A\left(\omega_{l}\right)=\bar{\omega}^{-1}$ is constant for $\omega_{l}>0$, the electric field corresponding to the above phase function can be written

$$
\begin{aligned}
\mathcal{E}(t)= & \operatorname{Re}\left\{\frac{1}{\bar{\omega}} \int_{0}^{\infty} e^{-i \omega_{l} t} d \omega_{l}\right. \\
& \left.-\frac{2}{\bar{\omega}_{n=1,3,5} \ldots} \int_{\Omega_{00}+n \omega-\varepsilon_{n}} e^{-i \omega_{l} t} d \omega_{l}\right\} \\
= & \operatorname{Re}\left\{e^{-i\left(\Omega_{00}+\omega\right) t} \frac{1}{\bar{\omega}_{n=-\infty}} \sum_{n}^{\infty}\left[\delta_{n 0}-2 \frac{\sin \left(\varepsilon_{n} t\right)}{\omega t}\right]\right. \\
& \left.\times\left[\pi \delta(t-n \pi / \omega)+i \mathcal{P}\left(\frac{1}{t-n \pi / \omega}\right)\right]\right\} .
\end{aligned}
$$

Note that the width of the function $\sin \left(\varepsilon_{n} t\right) / t$ is of the order of $2 \pi / \varepsilon_{n}$ and therefore larger than a vibrational period. The multipeaked structure of this field is quite similar to the result in Eq. (20) although the peaks here are spaced by half a vibrational period of the harmonic oscillator. Again all the peaks have the same functional form, except for a scale factor. 

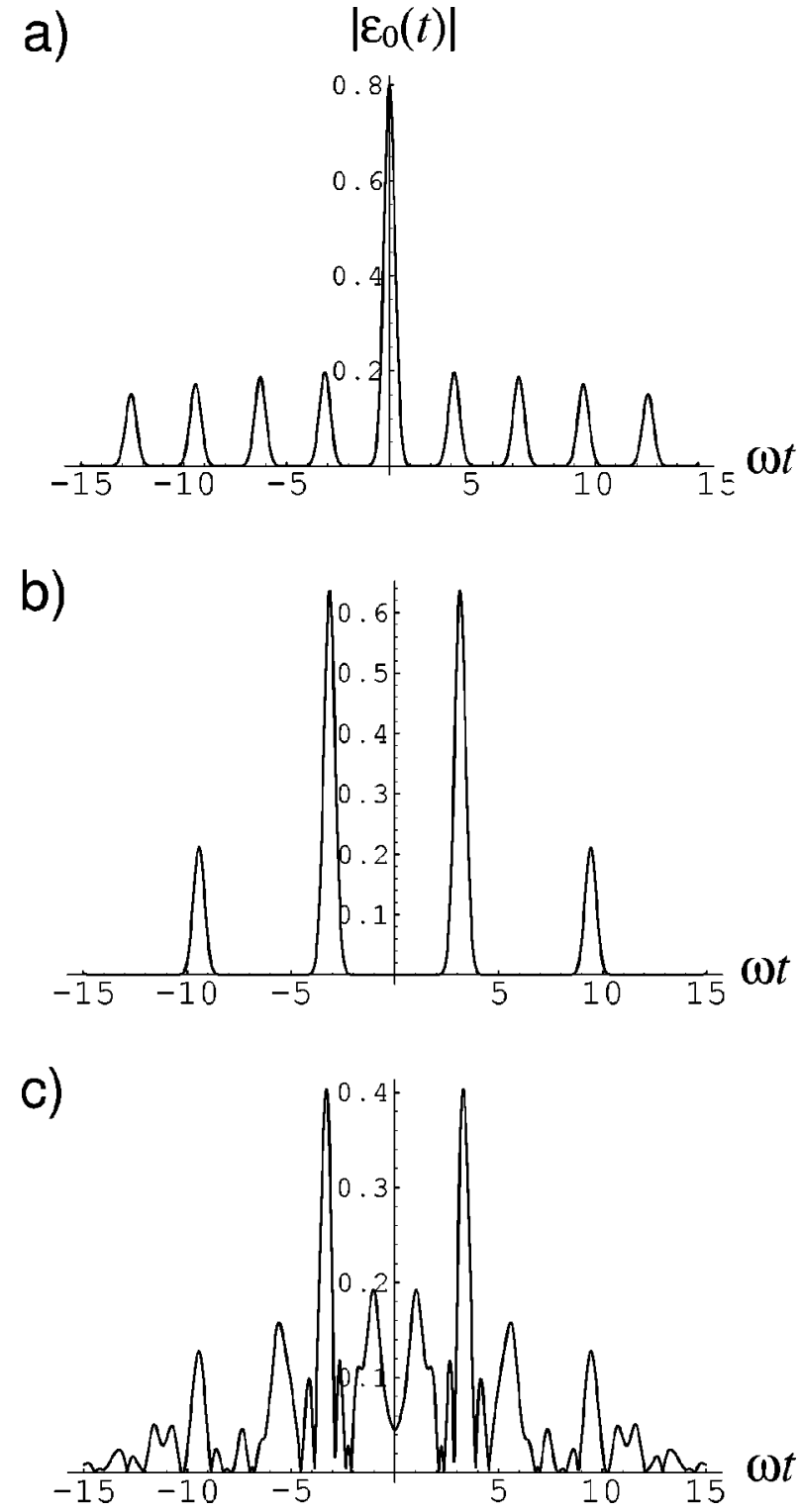

FIG. 3. The envelope of the electric field $\left|\mathcal{E}_{0}(t)\right|$ for a frequency distribution with a phase function given by segments with constant values of width $\varepsilon_{n}$, see Eq. (25), and a Gaussian frequency distribution with a temporal width (FWHM) of $\omega t=\pi / 5$. Panel (a) for $\varepsilon_{n}=0.1 \omega$, (b) for $\varepsilon_{n}=0.5 \omega$, and (c) for $\varepsilon_{n}$ chosen at random between 0 and $\omega$. Note that $\omega t=2 \pi$ corresponds to one vibrational period.

For a pulse with a Gaussian frequency distribution, $A\left(\omega_{l}\right)=\sqrt{a / \pi} \exp \left[-a\left(\omega_{l}-\omega_{0}\right)^{2}\right]$, with $A\left(\omega_{l}\right) \approx 0$ for $\omega_{l}$ $<\Omega_{00}$ (i.e., $\omega_{0}>\Omega_{00}+1 / \sqrt{a}$ ) the electric field of the pulse is obtained from Eq. (8),

$$
\begin{aligned}
\mathcal{E}(t)= & \pi e^{-t^{2} /(4 a)} \cos \left(\omega_{0} t\right) \\
& -2 \sum_{n=-\infty}^{\infty} \cos \left[\omega_{0} t-\pi n\left(\frac{\Delta}{\omega}+1\right)\right] \\
& \times \frac{\sin \left(n \pi \varepsilon_{n} / \omega\right)}{n} e^{-(t-n \pi / \omega)^{2} /(4 a)},
\end{aligned}
$$

where $\Delta=\omega_{0}-\Omega_{00}>1 / \sqrt{a}$.

Figure 3 shows the envelope $\left|\mathcal{E}_{0}(t)\right|$ of the electric field $\left(\mathcal{E}_{0}(t)\right.$ is the electric field where the fast oscillations due to the cosine terms have been omitted) for $a \omega^{2}=0.0355971$, corresponding to a (bandwidth-limited) Gaussian pulse where the pulse duration (FWHM) is $1 / 10$ of the vibrational period. Three different choices of the constant segments are illustrated: panel (a) $\varepsilon_{n}=0.1 \omega$, for all $n$; (b) $\varepsilon_{n}=0.5 \omega$, for all $n$; and (c) $\varepsilon_{n}$ chosen at random between 0 and $\omega$. All the results correspond to a multipeak structure of the field, and the temporal width of each peak in (a) and (b) is a direct reflection of the Gaussian frequency distribution. Note that from Eq. (27) with $\varepsilon \rightarrow 0$, we will obtain a result which corresponds to a choice of discrete and specific phases which are only specified at isolated frequencies. Figure 3(a) shows, however, that the convergence to this limit goes via an infinite series of pulses with vanishing amplitude, except for the Gaussian pulse centered around $t=0$.

\section{Trigonometric phase functions}

We consider here a periodic and even phase function, $\phi\left(\omega_{l}\right)=\pi\left[1-\cos \left(\pi \widetilde{\omega}_{l} / \omega\right)\right] / 2$ which oscillates periodically between zero and $\pi$. Note that $\exp \left[i \phi\left(\omega_{l}\right)\right]$ is periodic and complex. The Fourier transform is known analytically, ${ }^{23}$

$$
\begin{aligned}
& \int_{-\infty}^{\infty} e^{i \pi\left[1-\cos \left(\pi \omega_{l} / \omega\right)\right] / 2} e^{-i \omega_{l} t} d \omega_{l} \\
& \quad=i \sum_{n=-\infty}^{\infty}(-i)^{n} J_{n}(\pi / 2) \delta(t-n \pi / \omega),
\end{aligned}
$$

where $J_{n}(x)$ are Bessel functions of the first kind. We consider again a pulse with a Gaussian frequency distribution $A\left(\omega_{l}\right)=\sqrt{a / \pi} \exp \left[-a\left(\omega_{l}-\omega_{0}\right)^{2}\right]$, with the Fourier transform $\exp \left[-t^{2} /(4 a)\right] \exp \left(-i \omega_{0} t\right)$. The electric field of the pulse is again obtained from Eq. (8), using that the Fourier transform of a product of two functions is identical to the convolution of the individual transforms, ${ }^{23}$

$$
\begin{aligned}
\mathcal{E}(t)= & \sum_{n=-\infty}^{\infty} \cos \left[\omega_{0} t-\pi\left(\frac{n \Delta}{\omega}+\frac{1-n}{2}\right)\right] \\
& \times J_{n}(\pi / 2) e^{-(t-n \pi / \omega)^{2} /(4 a)} .
\end{aligned}
$$

Figure 4 shows the envelope $\left|\mathcal{E}_{0}(t)\right|$ of the electric field $\left[\mathcal{E}_{0}(t)\right.$ is the electric field where the fast oscillations due to the cosine term have been omitted] for $a \omega^{2}=0.0355971$, corresponding to a (bandwidth-limited) Gaussian pulse where the pulse duration (FWHM) is $1 / 10$ of the vibrational period. Clearly, the envelope contains again a series of peaks at $t=n \pi / \omega$, now with amplitudes given by the Bessel functions $J_{n}(\pi / 2)$.

We note that the Fourier transform of any periodic function $f\left(\omega_{l}\right)$ with the required period will give delta function samples $\delta(t-n \pi / \omega)$ weighted by the Fourier transform $F(t)$ of the function. ${ }^{23}$ Note that all our examples based on periodic phase functions, Eqs. (19), (20), (27), and (29), have this form. We get a multipeak structure of the field and all the peaks have the same functional form, except for a scale factor. Thus in this case one will always get a pulse duration of the order of, at least, one vibrational period. 


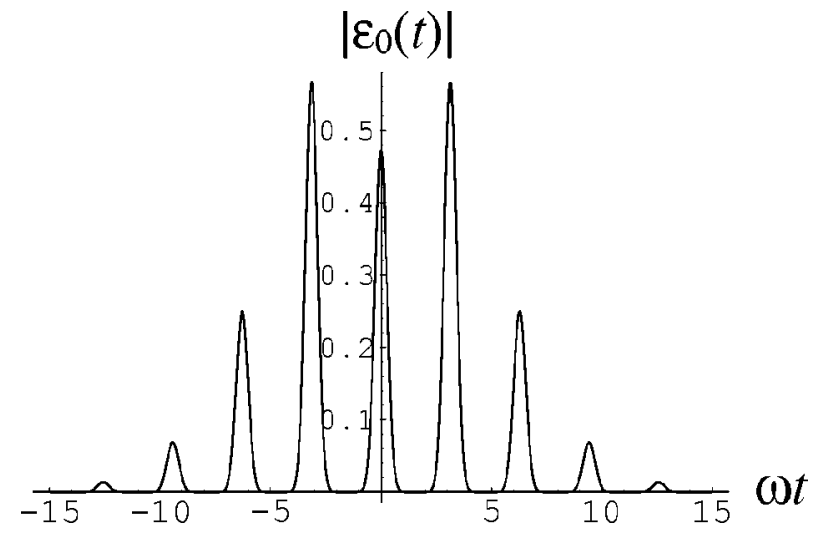

FIG. 4. The envelope of the electric field $\left|\mathcal{E}_{0}(t)\right|$ for a Gaussian frequency distribution with a periodic phase function given by $\phi\left(\omega_{l}\right)=\pi[1$ $\left.-\cos \left(\pi \widetilde{\omega}_{l} / \omega\right)\right] / 2$, where $\omega_{l}$ is the laser frequency, see Eq. (29). The temporal width (FWHM) of the transform limited Gaussian distribution is $\omega t$ $=\pi / 5$, which is also equal to the temporal width (FWHM) of the individual peaks. Note that $\omega t=2 \pi$ corresponds to one vibrational period.

\section{DISCUSSION AND CONCLUSIONS}

Experimentally, laser pulse shaping is accomplished via phase modulation of each spectral component in the frequency domain. The purpose of this work has been to investigate the possibilities/limitations of this approach; in particular, to understand the relation between a particular objective and the time it takes to create the desired target state, measured from the time the pulse is switched on. Given the experimental approach, it is natural to analyze the problem in the frequency/energy domain, however, at the expense that the time domain question is not directly addressed.

As an example, we have considered the possibility of instantaneous nonvertical electronic transitions and, in particular, the creation of a copy of the initial nuclear ground state at the outer turning point in bound excited-state potentials. Analyzing the problem in the frequency domain, the frequency dependence of the phases associated with each spectral component of the field must-for harmonic potentials - be chosen in a way which is equivalent to a time shift of the dynamics.

The problem we considered is how to implement the frequency dependence of the phases associated with each spectral component of the field, with specific phases required only at specific discrete excitation frequencies, such that the resulting field is ultrashort (on the time scale of nuclear motion). If successful, it would mean that a promoted state which coincides with the desired target state would be created using a pulse with a duration much less than half a vibrational period.

We presented investigations of various implementations of the frequency-dependent phase across the continuous frequency spectrum of the laser pulse. The introduction of a frequency-dependent phase function always resulted in a time shift and/or increased the temporal width of the laser pulse. One of the approaches that we considered was based on recent theoretical work on the construction of short pulse laser fields that will generate a desired target vibrational wave packet in a diatomic molecule. ${ }^{14}$ That approach failed in the present case. We also considered several other choices of the field, but the identification of a pulse that is nonzero only for times much smaller than half a vibrational period for a harmonic potential has been unsuccessful. Thus all the phase functions violate the assumption of an instantaneous excitation.

In fact, it is easy to see that it must be so if we switch to a time-domain description. Returning to Eqs. (1) and (2), we see that the excited-state wave function can be written in the form

$$
\chi_{2}(r, t)=\frac{i}{\hbar} \int_{0}^{\infty} d t^{\prime} e^{-i \epsilon_{0} t^{\prime} / \hbar} E\left(t^{\prime}\right) \phi\left(r, t-t^{\prime}\right),
$$

where $\phi\left(r, t-t^{\prime}\right)=\left\langle r\left|\exp \left[-i \hat{H}_{2}\left(t-t^{\prime}\right) / \hbar\right]\right| \phi\right\rangle$ and assuming, for convenience, that the laser field $E(t)$ is switched on for $t \geqslant 0$. Thus the excited-state wave function can be thought of as a coherent superposition of Franck-Condon wave packets promoted to the upper state at times $t^{\prime}$ with different weighting factors [given by $E\left(t^{\prime}\right)$ ] and phases. At time $t$, each of these wave packets in the superposition has evolved for at time $t-t^{\prime}$. Since it takes half a vibrational period for a Franck-Condon wave packet to reach the outer turning point, Eq. (30) shows that in order to create amplitude at the outer turning point, a time close to a half a vibrational period is required after the pulse was switched on.

As noted above, the present work is related to the time shifting concept which has recently been discussed and implemented experimentally. ${ }^{17}$ It was shown there that at early times one can create a wave packet that otherwise only would be obtained at longer times due to the natural dynamics of the system. In relation to that result, we have considered an extreme case and shown that there is a lower limit to this time shifting idea. That is, we cannot create a wave packet with amplitude at a given location faster than it takes a Franck-Condon wave packet to reach that location.

The result of this work is equivalent to the statement that it is not possible to move matter faster than the time associated with the natural (field-free) dynamics of the system. It must be emphasized that in the present paper we have only considered a weak-field description, based on first-order perturbation theory. Thus it could be relevant to investigate the question in the title of this paper also in the strong-field regime.

\section{ACKNOWLEDGMENTS}

This work was supported by the Danish Natural Science Research Council. We thank Jeff Krause for useful discussions.

${ }^{1}$ S. A. Rice and M. Zhao, Optical Control of Molecular Dynamics (Wiley, New York, 2000).

${ }^{2}$ P. Brumer and M. Shapiro, Annu. Rev. Phys. Chem. 43, 257 (1992).

${ }^{3}$ N. E. Henriksen, Chem. Soc. Rev. 31, 37 (2002).

${ }^{4}$ A. M. Weiner, D. E. Leaird, J. S. Patel, and J. R. Wullert, Opt. Lett. 15, 326 (1990).

${ }^{5}$ R. S. Judson and H. Rabitz, Phys. Rev. Lett. 68, 1500 (1992).

${ }^{6}$ A. Assion, T. Baumert, M. Bergt, T. Brixner, B. Kiefer, V. Seyfried, M. Strehle, and G. Gerber, Science 282, 919 (1998).

${ }^{7}$ T. C. Weinacht, J. Ahn, and P. H. Bucksbaum, Nature (London) 397, 233 (1999). 
${ }^{8}$ T. Brixner, N. H. Damrauer, P. Niklaus, and G. Gerber, Nature (London) 414, 57 (2001).

${ }^{9}$ R. J. Levis, G. M. Mekir, and H. Rabitz, Science 292, 709 (2001).

${ }^{10}$ D. Zeidler, S. Frey, K. L. Kompa, and M. Motzkus, Phys. Rev. A 64, 023420 (2001).

${ }^{11}$ J. L. Herek, W. Wohlleben, R. J. Cogdell, D. Zeidler, and M. Motzkus, Nature (London) 417, 533 (2002).

${ }^{12}$ D. Meshulach and Y. Silberberg, Nature (London) 396, 239 (1998).

${ }^{13}$ B. Kohler, V. V. Yakovlev, J. Che, J. L. Krause, M. Messina, K. R. Wilson, N. Schwentner, R. M. Whitnell, and Y. Yan, Phys. Rev. Lett. 74, 3360 (1995).

${ }^{14}$ L. E. E. de Araujo and I. A. Walmsley, J. Phys. Chem. A 103, 10409 (1999).
${ }^{15}$ E. J. Heller, Acc. Chem. Res. 14, 368 (1981).

${ }^{16}$ N. E. Henriksen, Adv. Chem. Phys. 91, 433 (1995).

${ }^{17}$ Z. Amitay, J. B. Ballard, H. U. Stauffer, and S. R. Leone, Chem. Phys. 267, 141 (2001)

${ }^{18}$ M. V. Rama Krishna and R. D. Coalson, Chem. Phys. 120, 327 (1988).

${ }^{19}$ J. Cao and K. R. Wilson, J. Chem. Phys. 106, 5062 (1997).

${ }^{20}$ V. S. Malinovsky and J. L. Krause, Chem. Phys. 267, 47 (2001).

${ }^{21}$ E. Merzbacher, Quantum Mechanics (Wiley, New York, 1970).

${ }^{22}$ J. Katriel, J. Phys. B 3, 1315 (1970).

${ }^{23}$ D. C. Champeney, Fourier Transforms and their Physical Applications (Academic, London, 1973). 